\title{
Combinational delivery of c-Myc siRNA and nucleoside analogues in a single, synthetic nanocarrier for targeted cancer therapy
}

\author{
Yuan Zhang ${ }^{1,2}$, Lei Peng ${ }^{1}$, Russell J Mumper ${ }^{1}$, and Leaf Huang ${ }^{1}{ }^{,}$ \\ ${ }^{1}$ Division of Molecular Pharmaceutics and Center for Nanotechnology in Drug Delivery, Eshelman \\ School of Pharmacy, University of North Carolina at Chapel Hill, Chapel Hill, NC 27599, USA
}

\begin{abstract}
The treatment of aggressive non-small-cell lung cancer (NSCLC) depends on the creation of new therapeutic regimens in clinical settings. In this study, we developed a Lipid/Calcium/Phosphate (LCP) nanoparticle that combines chemotherapy with gene therapy. By encapsulating a chemodrug, gemcitabine monophosphate (GMP), and siRNA specific to the undruggable c-Myc oncogene (c-Myc siRNA) into a single nanosized vesicle and systemically administering them to nude mice, we achieved potent anti-tumor activity in both subcutaneous and orthotopic models of NSCLC. The improvements in therapeutic response over either c-Myc siRNA or GMP therapy alone, were demonstrated by the ability to effectively induce the apoptosis of tumor cells and the significant reduction of proliferation of tumor cells. The combination therapy led to dramatic inhibition of tumor growth, with little in vivo toxicity. Additionally, the current studies demonstrated the possibility of incorporating both nucleic acid molecules and phosphorylated small molecule drugs into the inner core of a single nanoparticle formulation. Co-encapsulation of an oncogene-modulating siRNA and a chemotherapeutic agent will allow simultaneous interruption of diverse anti-cancer pathways, leading to increased therapeutic efficacy and reduced toxicities.
\end{abstract}

\section{Keywords}

c-Myc siRNA; Gemcitabine monophosphate; Calcium phosphate nanoparticle; Apoptosis; Proliferation

\section{Introduction}

Lung cancer continues to be the leading cause of cancer-related deaths both in the United States and throughout the world. Non-small-cell lung cancer (NSCLC) is the most

\footnotetext{
(C) 2013 Elsevier Ltd. All rights reserved.

* Corresponding author. Leaf Huang, Address: 1315 Kerr Hall CB\# 7571, Chapel Hill, NC 27599-7571, Telephone: 1-919-843-0736, Fax: 1-919-966-0197, leafh@unc.edu.

${ }^{2}$ Current address: Department of Materials Science and Engineering, Koch Institute for Integrative Cancer Research, Massachusetts Institute of Technology, Cambridge, MA 02139, USA

Publisher's Disclaimer: This is a PDF file of an unedited manuscript that has been accepted for publication. As a service to our customers we are providing this early version of the manuscript. The manuscript will undergo copyediting, typesetting, and review of the resulting proof before it is published in its final citable form. Please note that during the production process errors may be discovered which could affect the content, and all legal disclaimers that apply to the journal pertain.

Author contributions

Y.Z. performed all experiments, collected and analyzed the data and wrote the manuscript. L.P. and R.J.M. provided advice on the establishment of A549 lung orthotopic mouse model. L.H. supervised the project and contributed to writing the manuscript.
} 
aggressive type of lung cancer and accounts for up to $80-85 \%$ of all cases of lung cancer. Many NSCLC tumors over-express c-Myc, which is a pivotal transcription factor controlling the expressions of many proliferation genes, correlating with a poor prognosis for patients. $\mathrm{c}-\mathrm{Myc}$ is one of the most highly amplified, downstream oncogenes that promote cell growth, proliferation, invasion, expansion and angiogenesis.[1,2] Although the importance of c-Myc in oncogenesis and maintenance of the lung malignancy has long been recognized, no small molecule chemodrugs have been approved for clinical use. Thus, c-Myc remains an "undruggable" target, to which alternative approaches must be developed.[3] The advent of RNAi has brought about a fresh look in controlling any "undruggable" targets. Although siRNA is very potent and can specifically silence oncogenic targets, has to be protected from degradation and efficiently delivered to the cytoplasm of the tumor cells.

Antimetabolite gemcitabine (2', 2'-difluoro 2'-deoxycytidine) (Gem) is a nucleoside analogue that is activated intracellularly by phosphorylation, followed by incorporating into DNA to inhibit DNA synthesis, which leads to cell apoptosis.[4] The addition of the first phosphate group is the rate-limiting step in the nucleoside activation process.[5] GMP, the first activated species of Gem, can be efficiently phosphorylated to the bioactive derivative (i.e. gemcitabine triphosphate) by the cellular nucleoside kinase which is ubiquitous in mammalian cells.

The LCP nanoparticle is composed of a biodegradable and amorphous core of calcium phosphate $(\mathrm{CaP})$ precipitate that encapsulates therapeutic agents with a phosphate group $(\mathrm{s})$. The $\mathrm{CaP}$ core is then coated with an asymmetrical lipid bilayer. The inner leaflet, dioleoylphosphatydic acid (DOPA), is conjugated to the surface of the $\mathrm{CaP}$ cores through covalent bonds with the phosphate head group. The outer leaflet is composed of a cationic lipid, DOTAP, and a helper lipid, cholesterol. The membrane-coated, $\mathrm{CaP}$ core is further modified with a high density of polyethylene glycol phospholipid (DSPE-PEG) with a tethered targeting ligand anisamide (AA) grafted onto the surface of the nanoparticle for functionalization. AA is a small molecule that can specifically target the sigma receptors that are overexpressed by many human cancer cells. These characteristics of the nanoparticles allow a long circulation time in the blood, specific targeting to the tumor and evasion of the reticuloendothelial system.[6]

The calcium phosphate scaffold of the LCP described here could efficiently precipitate both nucleic acids and phosphorylated small molecules. In this study, we co-formulated both cMyc siRNA and GMP into a single LCP nanoparticle, and the therapeutic efficacy was tested in both subcutaneous and orthotopic xenograft models of human NSCLC.

\section{Materials and Methods}

\section{Materials}

Gemcitabine monophosphate disodium salt (GMP) was synthesized by HDH Pharma, Inc. (Research Triangle Park, NC). c-Myc siRNA (target sequence: 5'-AAC GUU AGC UUC ACC AAC AUU-3') and control siRNA (target sequence: 5'-AAU UCU CCG AAC GUG UCA CGU-3') were synthesized by Sigma-Aldrich (St. Louis, MO). 1, 2-Dioleoyl-3trimethylammonium-propane chloride salt (DOTAP), dioleoylphosphatydic acid (DOPA), and 1,2-distearoryl-sn-glycero-3-phosphoethanolamine- $\mathrm{N}$-[methoxy(polyethylene glycol-2000) ammonium salt (DSPE-PEG 2000 ) were purchased from Avanti Polar Lipids, Inc. (Alabaster, AL). DSPE-PEG-AA was synthesized in our lab as described in previous publications.[7] DeadEnd Fluorometric TUNEL assay kits were obtained from Promega Corporation (Madison, WI). Other chemicals were obtained from Sigma-Aldrich (St. Louis, $\mathrm{MO})$. 


\section{Cell culture}

H460 and A549 human NSCLC cells, originally obtained from American Type Culture Collection (ATCC), were cultured in an RPMI-1640 medium (Invitrogen, Carlsbad, CA) supplemented with $10 \%$ fetal bovine serum, $100 \mathrm{U} / \mathrm{mL}$ penicillin, and $100 \mu \mathrm{g} / \mathrm{mL}$ streptomycin (Invitrogen). Cells were cultivated in a humidified incubator at $37^{\circ} \mathrm{C}$ and $5 \%$ $\mathrm{CO}_{2}$. Cells were harvested with $0.05 \%$ trypsin-EDTA before subculture.

\section{Experimental animals}

Female nude mice that were 6-8 weeks of age were used in all studies. To establish the H460 xenograft models, $5 \times 10^{6} \mathrm{H} 460$ cells in $100 \mu \mathrm{L}$ of PBS were injected subcutaneously into the right flank of the mice. Experiments were performed 11 days after the mice were inoculated with $\mathrm{H} 460$ tumors. To create the A549 orthotopic model, nude mice were anaesthetized and a $0.5 \mathrm{~cm}$ incision was made after their skin was disinfected. A 28-gauge needle was used to inject $5 \times 10^{6}$ A549 cells in $40 \mu \mathrm{L}$ of Matrigel- PBS medium (v/v=1:1) approximately $2 \mathrm{~cm}$ above the bottom of the ribcage on the left dorsal side of nude mice. The incision was closed with a surgical clamp. All animals were maintained at a surgical plane of anesthesia during the procedure. Experiments were performed 4 weeks after the implantation of A549 tumors. The mice in the control group were untreated and received no injections. All work performed on animals was approved by the Institutional Animal Care and Use Committee at the University of North Carolina at Chapel Hill.

\section{Preparation of CMyc-LCP-AA, GMP-LCP-AA and (GMP+CMyc)-LCP-AA}

LCP cores were prepared using water-in-oil micro-emulsions, with the oil phase containing cyclohexane/ Igepal CO-520 solution (71/29, v/v).[8]. To prepare the cores of cMyc-LCP, $48 \mu \mathrm{g}$ c-Myc siRNA was mixed with $600 \mu \mathrm{L} 2.5 \mathrm{M} \mathrm{CaCl}_{2}$ and added into $20 \mathrm{~mL}$ of oil phase, where the other emulsion contained $600 \mu \mathrm{L} 12.5 \mathrm{mM} \mathrm{Na}_{2} \mathrm{HPO}_{4}(\mathrm{pH}=9.0)$. The GMPLCP core was formulated using $90 \mu \mathrm{L}$ of $60 \mathrm{mM}$ GMP mixed with $12.5 \mathrm{mM} \mathrm{Na}_{2} \mathrm{HPO}_{4}$ $(\mathrm{pH}=9.0)$ (final concentration) to reach a total volume of $600 \mu \mathrm{L}$. This solution was added into $20 \mathrm{~mL}$ of oil phase. Simultaneously, six-hundred $\mu \mathrm{L}$ of $2.5 \mathrm{M} \mathrm{CaCl}_{2}$ was added to a separate $20 \mathrm{~mL}$ oil phase. To prepare the cores of (GMP+cMyc)-LCPs, the phosphate phase met the same specifications outlined in the preparation of the cores of the GMP-LCP. The calcium phase contained $600 \mu \mathrm{L}$ of $2.5 \mathrm{M} \mathrm{CaCl}_{2}$ mixed with $48 \mu \mathrm{g}$ of c-Myc siRNA. Fourhundred $\mu \mathrm{L}$ of $20 \mathrm{mM}$ DOPA in chloroform was added to the phosphate phase of the GMP$\mathrm{LCP}$ and (GMP+cMyc)-LCP, whereas $200 \mu \mathrm{L}$ of $20 \mathrm{mM}$ DOPA was added to the phosphate phase during the preparation of the $\mathrm{cMyc}-\mathrm{LCP}$. The two separate micro-emulsions were then mixed and stirred for $5 \mathrm{~min}$. Another $400 \mu \mathrm{L}$ of $20 \mathrm{mM}$ DOPA was added into the emulsion of GMP-LCP and (GMP+cMyc)-LCP; $200 \mu \mathrm{L}$ of $20 \mathrm{mM}$ DOPA was added to cMyc-LCP. The emulsion was continually stirred for another $20 \mathrm{~min}$ before $40 \mathrm{~mL}$ of absolute ethanol was added. The ethanol emulsion was centrifuged at 10,000 $\mathrm{g}$ for $15 \mathrm{~min}$ to pellet the LCP cores and the supernatant was then discarded. The LCP cores were washed twice with absolute ethanol and dried under $\mathrm{N}_{2}$. The LCP core pellets were suspended in $2 \mathrm{~mL}$ chloroform and stored in a glass vial at $-20^{\circ} \mathrm{C}$.

To prepare the final cMyc-LCP-AA, GMP-LCP-AA and (GMP+cMyc)-LCP-AA with outer lipid coating, $330 \mu \mathrm{L}$ of the LCP cores in chloroform was mixed with $38.7 \mu \mathrm{L}$ of $10 \mathrm{mg} / \mathrm{mL}$ Cholesterol, $28 \mu \mathrm{L}$ of $25 \mathrm{mg} / \mathrm{mL}$ DOTAP, $76.8 \mu \mathrm{L}$ of $25 \mathrm{mg} / \mathrm{mL}$ DSPE-PEG and $19.2 \mu \mathrm{L}$ of $25 \mathrm{mg} / \mathrm{mL}$ DSPE-PEG-AA. After evaporating the chloroform, the residual lipids were dissolved in $30 \mu \mathrm{L}$ THF followed by $50 \mu \mathrm{L}$ absolute ethanol, and then suspended in $160 \mu \mathrm{L}$ water. After sonication, the solution was dialyzed in distilled water to remove the THF and ethanol. The preparation procedure of (CMP+Con)-LCP-AA was identical to that of (GMP $+\mathrm{cMyc}$ )-LCP-AA, except that GMP and c-Myc siRNA were replaced by equal molar amounts of CMP and control siRNA. 


\section{Characterization of cMyc-LCP-AA, GMP-LCP-AA and (GMP+cMyc)-LCP-AA}

The particle size and zeta potential of LCPs were determined using dynamic light scattering performed with a Malvern ZetaSizer Nano series (Westborough, MA). The encapsulation efficiency (EE\%) of GMP or siRNA was measured after lysing the LCPs with a THF/1M $\mathrm{HCl}(\mathrm{v} / \mathrm{v}=70 / 30)$ solution. GMP EE\% was measured using a UV spectrophotometer (Beckman Coulter Inc., DU 800 spectrophotometer) at a wavelength of $275 \mathrm{~nm}$. The EE\% of siRNA was measured by mixing a small amount of Tritium labeled oligonucleotide with cMyc siRNA in the LCP cores. Tritium-labeled oligonucleotide served as a marker for the entrapped siRNA; the radioactivity of the tritium was measured using a liquid scintillation analyzer (Packard BioScience Co. TRICARB 2900TR) and the percentage of siRNA entrapped in the cores of LCP was calculated. TEM images of LCP formulations were acquired through the use of JEOL 100CX II TEM (Tokyo, Japan). Over 2 min, $4 \mu$ of LCP solution was dropped onto a carbon-coated copper grid (Ted Pella, Inc., Redding, CA). Excess fluid was then removed with filter paper and the copper grid was dried before performing observation using the TEM.[9]

\section{In vitro cytotoxicity assay}

In vitro cytotoxicity of different LCPs and free GMP was determined using a 3-[4, 5dimethylthiazol-2-yl]-2, 5-diphenyltetrazolium bromide (MTT) assay. H460 and A549 cells were seeded at a density of $1 \times 10^{4}$ cells per well in 96 -well plates. When cells reached $30 \%$ confluence, different concentrations of cMyc-LCP-AA, GMP-LCP-AA, (GMP+cMyc)LCP-AA, (CMP+Con)-LCP-AA, GMP-LCP-AA and cMyc-LCP-AA mixtures (each at half dose) as well as free GMP were added to the medium. The dose of LCP was based on the dose of GMP and the doses of other LCPs were calculated using the lipid concentration that was equivalent to the corresponding doses of GMP-LCP-AA. After $48 \mathrm{~h}$ of exposure to the LCPs and free GMP at $37^{\circ} \mathrm{C}$, MTT (Biosynth Inc.) was added to each well to achieve a final concentration of $0.5 \mathrm{mg} / \mathrm{mL}$. The cells were then incubated with the MTT at $37^{\circ} \mathrm{C}$ for $4 \mathrm{~h}$. The resulting formazan crystals were solubilized by adding $150 \mu \mathrm{L}$ DMSO to each well. The absorbance was measured using a microplate reader at a wavelength of $570 \mathrm{~nm}$. Cell viability was calculated as the percentage of the absorbance of the treated cells to that of untreated cells.

\section{Western blot analysis}

Mice bearing H460 tumors were given daily IV injections for three days. Twenty-four h after the third injection, mice were sacrificed and tumor lysates were prepared for western blot analysis using the previously described method.[4] The mouse monoclonal c-Myc and mouse monoclonal poly(ADP-ribose) polymerase-1 (PARP-1) (1:500 dilution; Santa Cruz Biotechnology) antibodies were probed on the PVDF membrane overnight at $4{ }^{\circ} \mathrm{C}$. $\beta$-actin antibodies (1:4000 dilution; Santa Cruz Biotechnology) served as the loading control.

\section{Immunostaining}

One day after three daily IV injections, mice bearing H460 tumors or implanted with A549 orthotopic tumors were sacrificed and tumors were fixed in $10 \%$ formalin for $24 \mathrm{~h}$ before they were embedded in paraffin and sectioned at a thickness of $5 \mu \mathrm{m}$. The TUNEL staining was performed as recommended by the manufacturer (Promega, Madison, WI). DAPI mounting medium was then used to stain the nuclei. The percentage of apoptotic cells in the samples was obtained by dividing the number of apoptotic cells (TUNEL positive cells shown as green dots) from the number of total cells (blue nuclei stained by DAPI, not shown) in each microscopic field, and 10 representative microscopic fields were randomly selected in each treatment group for this analysis. 
Proliferation of tumor cells in H460 and A549 xenografts after the aforementioned treatments was determined using immunohistochemistry, using an antibody against proliferating cell nuclear antigen (PCNA) (1:200 dilution, Santa Cruz). The immunohistochemistry was performed using a mouse-specific, HRP/DAB detection IHC kit as recommended by the manufacturer (Abcam, Cambridge, MA). The percentage of cells that were proliferating was obtained by dividing the number of PCNA positive cells (shown as brown dots) by the number of total cells (blue nuclei stained by hematoxylin) in each microscopic field. Ten microscopic fields were randomly selected to represent each treatment group for counting.

\section{Tumor growth inhibition}

The inhibition of tumor growth in an $\mathrm{H} 460$ xenograft model was determined. When the tumor volumes reached $150-200 \mathrm{~mm}^{3}$, mice were randomly assigned into 6 treatment groups, and intravenously injected with different LCPs, including cMyc-LCP-AA, GMPLCP-AA, (GMP+cMyc)-LCP-AA, (CMP+Con)-LCP-AA as well as GMP-LCP-AA and cMyc-LCP-AA mixtures (each at half dose). The IV injections were given every other day for a total of 4 injections at a dose of $25 \mu \mathrm{mol} / \mathrm{Kg}$ GMP and/or $0.2 \mathrm{mg} / \mathrm{Kg}$ c-Myc siRNA. Tumor sizes were measured every other day with calipers across two perpendicular diameters; volume was calculated using the following formula: $\mathrm{V}=0.5 \times\left(\mathrm{W}^{2} \times \mathrm{L}\right)$, where $\mathrm{V}=$ tumor volume, $\mathrm{W}=$ the smaller perpendicular diameter and $\mathrm{L}=$ the larger perpendicular diameter. Two days after the final injection, the mice were sacrificed and the tumors were stripped off.

\section{In vivo toxicity}

BALB/c mice were given three daily IV injections of different LCPs. Twenty-four $h$ after the final injection, blood was drawn from the venous plexus of the eyes of the mice. Blood samples were centrifuged at $6,000 \mathrm{~g}$ and $4^{\circ} \mathrm{C}$ f or $15 \mathrm{~min}$, and the supernatant blood serums were collected for hematological analysis. Blood urine nitrogen (BUN), creatinine, aspartate aminotransferase (AST), and alanine aminotransferase (ALT) values were recorded as indications of hepatic and renal function. Organs (heart, liver, spleen, lung, kidney) of mice in different treatment groups were fixed and sectioned for H\&E staining.

\section{Statistical analysis}

Results were expressed as means \pm standard deviation (SD). Student t-tests were used to evaluate statistical significance. A result of $p<0.05$ was considered to be statistically significant.

\section{Results}

\section{Characterization of drug-loaded LCPs}

The photographs obtained from the TEM showed that all drug-loaded LCP-AAs had a spherical shape, and were monodispersed with approximately $20 \mathrm{~nm}$ in diameter (Fig. 1). The zeta potentials of cMyc-LCP-AA, GMP-LCP-AA and (GMP+cMyc)-LCP-AA were $33.3 \pm 7.1 \mathrm{mV}, 9.1 \pm 5.4 \mathrm{mV}$, and $28.9 \pm 4.5 \mathrm{mV}$, respectively. The EE\% of c-Myc siRNA was $55 \%$ and of GMP in LCP-AA was 75\%. The EE\% of c-Myc siRNA and GMP in coformulated LCP-AA was almost the same as that of the cMyc-LCP-AA or GMP-LCP-AA single formulation, which indicates that c-Myc siRNA and GMP did not interfere with each other in the process of co-precipitation with calcium ions within the LCP cores. In other words, the phosphate backbone of the nucleic acid molecules interact with calcium in a way that is similar to that of phosphorylated, hydrophilic small molecules. 


\section{Cell viabilities of drug-loaded LCPs}

The MTT assay was carried out to determine the cell viabilities after the treatment of different drug-loaded LCPs and free GMP. After $48 \mathrm{~h}$ incubation, GMP-LCP-AA, (GMP $+\mathrm{cMyc}$ )-LCP-AA and free GMP caused a dramatic reduction of viable H460 (Fig. 2A) and A549 (Fig. 2B) cells in a dose-dependent manner. LCPs enter into cells through sigma receptor-mediated endocytosis, while free GMP can be metabolized to gemcitabine before entering into cells through nucleoside transporters.[4] cMyc-LCP-AA showed limited cytotoxicity. GMP-LCP-AA and the cMyc-LCP-AA mixture (each at half dose) showed partial cytotoxicity at medium dose range, and the cell viabilities decreased at high doses. The cytotoxicity is mainly caused by encapsulated GMP rather than c-Myc siRNA. Additionally, $\mathrm{H} 460$ cells were more sensitive to the GMP than A549 cells. The $\mathrm{IC}_{50}$ values of A549 cells were about three times as much as that of $\mathrm{H} 460$ cells. (CMP+Con)-LCP-AA had little effect on cell viability, and the slight cytotoxicity shown at the high dose was due to the relatively high concentration of DOTAP cationic lipids in the cell culture medium. [10]

\section{c-Myc down-regulation and apoptosis in vivo}

Mice subcutaneously bearing H460 tumors were given three daily IV injections of different LCP formulations at a dose of $25 \mu \mathrm{mol} / \mathrm{Kg}$ GMP $(9.7 \mathrm{mg} / \mathrm{Kg}$ GMP, or $6.6 \mathrm{mg} / \mathrm{Kg}$ in terms of Gem) and/or $0.2 \mathrm{mg} / \mathrm{Kg}$ c-Myc siRNA. Twenty-four $\mathrm{h}$ after the third injection, mice were sacrificed and tumor lysates were prepared for western blot analysis. cMyc-LCP-AA and (GMP+cMyc)-LCP-AA displayed dramatic c-Myc down-regulation, while the GMP-LCPAA and particle mixture (each at half dose) showed partial effects (Fig. 3). Our results indicated that chemotherapeutic GMP limited the c-Myc expression in NSCLC after multiple administrations, probably through the inhibition of tumor cell survival and proliferation.[11] PARP is a nuclear protein that performs central roles in the repair of damaged DNA and is involved in the programmed cell death.[12] cMyc-LCP-AA overexpressed the intact PARP. GMP-LCP-AA, (GMP+cMyc)-LCP-AA and the particle mixture further stimulated the PARP cleavage, suggesting an advanced stage of apoptotic induction.[13] Compared to the control, (CMP+Con)-LCP-AA had no measurable effect on the protein expression level of c-Myc and PARP (Fig. 3).

\section{Tumor cell apoptosis in vivo}

We measured apoptosis in tumor tissues in H460 subcutaneous and A549 orthotopic xenografts using the TdT-mediated dUTP Nick-End Labeling (TUNEL) assay. Twenty-four $\mathrm{h}$ after three daily IV injections, tumor tissues were collected and sectioned for analysis of apoptosis. In subcutaneous xenografts of $\mathrm{H} 460$, (GMP+cMyc)-LCP-AA triggered apoptosis in $\sim 28 \%$ of cells. GMP-LCP-AA and cMyc-LCP-AA led to $\sim 23$ and $\sim 3 \%$ apoptotic tumor cells, respectively. Compared to formulations of single agents, (GMP+cMyc)-LCP-AA showed an additive effect. In A549 orthotopic xenografts, (GMP+cMyc)-LCP-AA triggered a dramatic killing effect, inducing apoptosis $\sim 46 \%$ of the lung tumor cells, while GMP-LCPAA and cMyc-LCP-AA led to 12\% apoptotic tumor cells. Thus, in an A549 orthotopic model, (GMP+cMyc)-LCP-AA displayed a synergistic effect compared to either formulation alone. Since A549 cells were not as sensitive to GMP as H460 cells (Fig. 2), the combined therapeutic strategy was shown to be more effective than chemotherapy alone in both xenograft models. (CMP+Con)-LCP-AA did not elicit apoptosis in tumor cells in either xenograft model (Fig. 4A and 4C).

\section{Tumor cell proliferation in vivo}

We also evaluated the effect of different LCP formulations on the proliferation of $\mathrm{H} 460$ and A549 xenografts. PCNA is expressed in the cell nuclei during DNA synthesis and can be 
used as a marker for cell proliferation. As shown in Fig. 5, cMyc-LCP-AA and (GMP + cMyc)-LCP-AA significantly decreased the number of PCNA positive cells in tumors. The GMP-LCP-AA and nanoparticle mixture (each at half dose) also caused reductions in the proliferation of tumor cells, but not as potent as cMyc-LCP-AA and (GMP+cMyc)-LCPAA. Compared to GMP, the down-regulation of c-Myc in NSCLC was more effective in inhibiting tumor cell proliferation. c-Myc is critically involved in the regulation of many growth-promoting signaling pathways and has been widely characterized as a driver of cell proliferation.[14] (CMP+Con)-LCP-AA and free GMP showed little anti-proliferative effect.

\section{Tumor growth inhibition}

The inhibition of tumor growth was evaluated in H460 subcutaneous model. H460 tumorbearing mice were treated intravenously every other day for a total of 4 injections at a dose of $25 \mu \mathrm{mol} / \mathrm{Kg}$ GMP and/or $0.2 \mathrm{mg} / \mathrm{Kg}$ c-Myc siRNA. As shown in Fig. 6A, (GMP+cMyc)LCP-AA exhibited the most effective inhibition of tumor growth. The GMP-LCP-AA, cMyc-LCP-AA and nanoparticle mixtures also effectively suppressed tumor growth, but not as potently as the combined nanoparticle treatment. The (CMP+Con)-LCP-AA had little effect on the inhibition of tumor growth compared to the control. At the treatment end point, tumors in each treatment group were harvested and the weight of the tumors was measured (Fig. 6B). Mice treated with (GMP+cMyc)-LCP-AA had tumor weights that were significantly less than those of the control. The tumor weights in groups treated with GMPLCP-AA, cMyc-LCP-AA and nanoparticle mixtures were at least twice those of the weights of the tumors of the group receiving the combined therapy. Thus, the combined treatment with (GMP+cMyc)-LCP-AA was more effective than treatment with GMP-LCP-AA and cMyc-LCP-AA individually. No serious toxicity was observed, as indicated by body weight changes (Fig. 6C).

\section{In vivo toxicities}

To test whether LCPs loaded with GMP and c-Myc siRNA would induce in vivo toxicity, especially hepatic and renal dysfunction after frequent multiple dosing, BALB/c mice were given daily IV injections for three days. Twenty-four $h$ after the final injection, blood serum samples were obtained for hematological analysis and the histopathology of different organs was evaluated using H\&E staining. Liver enzymes and renal indicators were measured. As shown in Table 1, there was little elevation of BUN, creatinine and AST, ALT in blood serum compared to the control; all parameter values were in the normal range. Thus, drugloaded LCPs administered at the therapeutic dose did not elicit any toxicity in the kidney or liver and there were no significant safety concerns. The administration of chemotherapy to patients with liver impairment may result in complicated safety issues. Therefore, a treatment regimen using the nanoparticles formulated with both GMP and c-Myc siRNA can help alleviate the potential liver toxicity as well as enhance the therapeutic response. From the H\&E-stained tissue sections of heart, liver, spleen, lung and kidney (Supplementary Fig. 1), we determined that there were no noticeable histological changes between the control groups and those treated with drug-loaded LCP, which showed no evidence of organ toxicity. Taken together, LCP formulations encapsulating chemotherapeutic agents and therapeutic siRNAs are safe for in vivo applications.

\section{Discussion}

This study investigates the feasibility of integrating nanotechnology with combination therapy. Combined therapy of two or more drugs promotes synergism and suppresses the progression of tumors through distinct mechanisms of action. LCP nanoparticle delivery platform could concurrently deliver multiple phosphorylated chemodrugs and gene 
therapeutics to the target site, enhancing therapeutic efficacy and reducing side effects of the drug payloads. Administrating multiple chemo- and gene- therapeutics with different molecular targets can raise the genetic barriers that need to be overcome for cancer cell mutations,[15] thereby alleviating the development of drug resistance. There are now a number of active chemotherapeutic regimens developed for NSCLC, but these chemotherapeutic treatments provide only modest extensions of life and do not provide curative treatment. In recent years, significant efforts have been made to identify agents (e.g., small molecules or biological agents such as antibodies) that selectively target cancer cells and/or genes that are expressed mainly in cancer-associated pathways.

While targeted molecular therapy is attractive, there are still many lung cancer patients for which the precise molecular mutation(s) involved in their disease cannot be determined. Gemcitabine (Gemzarß) is a nucleoside analogue of cytosine and it is commonly used chemotherapeutic drug, especially for non-small-cell lung carcinoma. Gem is transported into mammalian cells via the nucleoside transporter and phosphorylated into mono-, di- and tri-nucleotides inside the cells. Gemcitabine triphosphate then enters the nucleus and is incorporated into cellular DNA during the $\mathrm{S}$ phase of the cell cycle, blocking the replication of DNA.[4] Thus, Gem is actually a prodrug that requires further cellular metabolism for its activity. Furthermore, pharmacokinetics of Gem indicate that it is rapidly deaminated in the blood, becoming inactive difluorouridine, after which it is cleared from the blood through renal excretion.[16] Nucleoside analogues, such as GMP, have limited uses as drugs because they are not transported by the nucleoside transporter. Using a nanocarrier to protect and deliver more Gem derivatives to the tumor is an excellent strategy for enhancing the efficacy of the drug, while also reducing the toxicity.

The c-Myc oncogene is overexpressed in numerous tumors, such as lung, breast, pancreatic, gallbladder and colon carcinomas, where its downstream target genes promote cell growth, proliferation, invasion, expansion and angiogenesis.[1,2,17] Despite being one of the earliest oncogenes identified, the c-Myc transcription factor remains "undruggable" by small molecules and/or antibodies for a variety of reasons. For example, the basic, helix-loop-helix structure of the transcription factor protein of c-Myc does not possess drug-accessible domains. Furthermore, as with other transcription factors, c-Myc carries out a multitude of physiological functions and its prolonged down-regulation can adversely affect healthy cells. [18] Nevertheless, c-Myc represents a promising target for siRNA-mediated downregulation of proteins; several reports demonstrate the feasibility of this approach in inhibiting tumor growth in multiple cancer models.[19, 20] Selective oncogene silencing via siRNA has shown great promise for cancer therapy as these have been shown to inhibit the mRNA synthesis that can lead to down-regulation of the cancer associated protein.[21]

We choose to use c-Myc siRNA as a viable and sound "undruggable" candidate in combination with the derivatives of a conventional chemotherapeutic agent Gem. Codelivery of c-Myc siRNA and GMP will obstruct the progression of tumor cells through two independent pathways. c-Myc siRNA and GMP can be co-entrapped in AA ligand-targeted LCP nanoparticles, delivered across the membranes of the tumor cells in vivo through sigma receptor-mediated endocytosis. The $\mathrm{CaP}$ cores of the LCPs are dissolvable in endosomes due to their low $\mathrm{pH}$. The increased osmotic pressure in the endosome leads to endosome swelling and rupture, releasing the entrapped c-Myc siRNA and GMP into the cytoplasm. The cationic lipid DOTAP wrapping the LCP core may also promote the release of the entrapped cargos from endosomes into the cytosol by destabilizing the anionic membranes of the endosomes.[22] Once escaped from the endosomes, c-Myc siRNA guides the sequence-specific degradation of mRNA through RNA interference machinery, leading to down-regulation of the c-Myc protein. The released GMP, after further phosphorylation, 
simultaneously diffuses from the cytoplasm into the nucleus where it can induce apoptosis through the disruption of the transcription mechanism of cellular DNA.

Nucleic acids can potentially be combined with chemotherapeutics in the LCP delivery vehicle to strategically disrupt the progression of tumors at the level of protein (siRNA) and DNA synthesis (chemotherapeutics). This ability enables our nanoparticle-based co-delivery to be an efficient therapeutic tool for the coordinated treatment of cancer, simultaneously targeting unique points in the cancer signaling cascade. Taken together, the development of a single nanoparticle formulation with multifunctional anti-cancer activity can overcome their individual innate limitations, and expand the efficacy of each mono-therapeutic regimen, leading to superior therapeutic responses.

\section{Conclusion}

In the present study, we successfully encapsulated chemotherapeutic agent GMP and siRNA targeting to c-Myc into a single, targeted LCP nanoparticle. We have shown that the combined chemo- and gene- therapy with $(\mathrm{GMP}+\mathrm{cMyc})-\mathrm{LCP}-\mathrm{AA}$ can induce tumor cell apoptosis and inhibit tumor cell proliferation effectively in both subcutaneous and orthotopic models of NSCLC. The combined therapeutic regimen significantly delayed the tumor progression, with little in vivo toxicity. The LCP nanotechnology allows the coencapsulation of chemotherapeutics and gene therapeutics into a single, synthetic nanocarrier, and simultaneously targets diverse anti-cancer pathways in a coordinated fashion, leading to increased therapeutic efficacy and reduced toxicities.

\section{Supplementary Material}

Refer to Web version on PubMed Central for supplementary material.

\section{Acknowledgments}

We thank Kelly Racette for editing the manuscript. Research was supported by NIH grants CA151652 and CA149363.

\section{References}

1. de Nigris F, Balestrieri ML, Napoli C. Targeting c-Myc, Ras and IGF cascade to treat cancer and vascular disorders. Cell Cycle. 2006; 5(15):1621-1628. [PubMed: 16921263]

2. Dang CV. MYC on the path to cancer. Cell. 2012; 149(1):22-35. [PubMed: 22464321]

3. Toyoshima M, Howie HL, Imakura M, Walsh RM, Annis JE, Chang AN, et al. Functional genomics identifies therapeutic targets for MYC-driven cancer. Proc Natl Acad Sci U S A. 2012; 109(24): 9545-9550. [PubMed: 22623531]

4. Zhang Y, Kim WY, Huang L. Systemic delivery of gemcitabine triphosphate via LCP nanoparticles for NSCLC and pancreatic cancer therapy. Biomaterials. 2013; 34(13):3447-3458. [PubMed: 23380359]

5. Oguri T, Achiwa H, Sato S, Bseeho Y, Takano Y, Miyazaki M, et al. The determinants of sensitivity and acquired resistance to gemcitabine differ in non-small cell lung cancer: a role of ABCC5 in gemcitabine sensitivity. Mol Cancer Ther. 2006; 5(7):1800-1806. [PubMed: 16891466]

6. Zhang Y, Satterlee A, Huang L. In vivo gene delivery by nonviral vectors: overcoming hurdles? Mol Ther. 2012; 20(7):1298-1304. [PubMed: 22525514]

7. Banerjee R, Tyagi P, Li S, Huang L. Anisamide-targeted stealth liposomes: a potent carrier for targeting doxorubicin to human prostate cancer cells. Int J Cancer. 2004; 112(4):693-700. [PubMed: 15382053]

8. Li J, Yang Y, Huang L. Calcium phosphate nanoparticles with an asymmetric lipid bilayer coating for siRNA delivery to the tumor. J Control Release. 2012; 158(1):108-114. [PubMed: 22056915] 
9. Zhang Y, Wang X, Wang J, Zhang X, Zhang Q. Octreotide-modified polymeric micelles as potential carriers for targeted docetaxel delivery to somatostatin receptor overexpressing tumor cells. Pharm Res. 2011; 28(5):1167-1178. [PubMed: 21340573]

10. Lv H, Zhang S, Wang B, Cui S, Yan J. Toxicity of cationic lipids and cationic polymers in gene delivery. J Control Release. 2006; 114(1):100-109. [PubMed: 16831482]

11. Gong HT, Sun L, Ren XJ, Liu L, Han XF. Influence of gemcitabine on expression of C-myc gene and its apoptosis-inducing effect on HL-60 cells. Zhongguo Shi Yan Xue Ye Xue Za Zhi. 2010; 18(2):317-320. [PubMed: 20416159]

12. Chaitanya GV, Steven AJ, Babu PP. PARP-1 cleavage fragments: signatures of cell-death proteases in neurodegeneration. Cell Commun Signal. 2010; 8:31. [PubMed: 21176168]

13. Boulares AH, Yakovlev AG, Ivanova V, Stoica BA, Wang G, Lyer S, et al. Role of poly(ADPribose) polymerase (PARP) cleavage in apoptosis. Caspase 3-resistant PARP mutant increases rates of apoptosis in transfected cells. J Biol Chem. 1999; 274(33):22932-22940. [PubMed: 10438458]

14. Miller DM, Thomas SD, Islam A, Muench D, Sedoris K. c-Myc and cancer metabolism. Clin Cancer Res. 2012; 18(20):5546-5553. [PubMed: 23071356]

15. Hu CM, Aryal S, Zhang L. Nanoparticle-assisted combination therapies for effective cancer treatment. Ther Deliv. 2010; 1(2):323-334. [PubMed: 22816135]

16. Shipley LA, Brown TJ, Cornpropst JD, Hamilton M, Daniels WD, Culp HW. Metabolism and disposition of gemcitabine, and oncolytic deoxycytidine analog, in mice, rats, and dogs. Drug Metab Dispos. 1992; 20(6):849-855. [PubMed: 1362937]

17. Baudino TA, McKay C, Pendeville-Samain H, Nilsson JA, Maclean KH, White EL, et al. c-Myc is essential for vasculogenesis and angiogenesis during development and tumor progression. Genes Dev. 2002; 16(19):2530-2543. [PubMed: 12368264]

18. Soucek L, Evan GI. The ups and downs of Myc biology. Curr Opin Genet Dev. 2010; 20(1):91-95. [PubMed: 19962879]

19. Kabilova TO, Chernolovskaya EL, Vladimirova AV, Vlassov VV. Inhibition of human carcinoma and neuroblastoma cell proliferation by anti-c-myc siRNA. Oligonucleotides. 2006; 16(1):15-25. [PubMed: 16584292]

20. Wang YH, Liu S, Zhang G, Zhou CQ, Zhu HX, Zhou XB, et al. Knockdown of c-Myc expression by RNAi inhibits MCF-7 breast tumor cells growth in vitro and in vivo. Breast Cancer Res. 2005; 7(2):R220-R228. [PubMed: 15743499]

21. Wang Z, Rao DD, Senzer N, Nemunaitis J. RNA interference and cancer therapy. Pharm Res. 2011; 28(12):2983-2995. [PubMed: 22009588]

22. Xu Y, Szoka FC Jr. Mechanism of DNA release from cationic liposome/DNA complexes used in cell transfection. Biochemistry. 1996; 35(18):5616-5623. [PubMed: 8639519] 

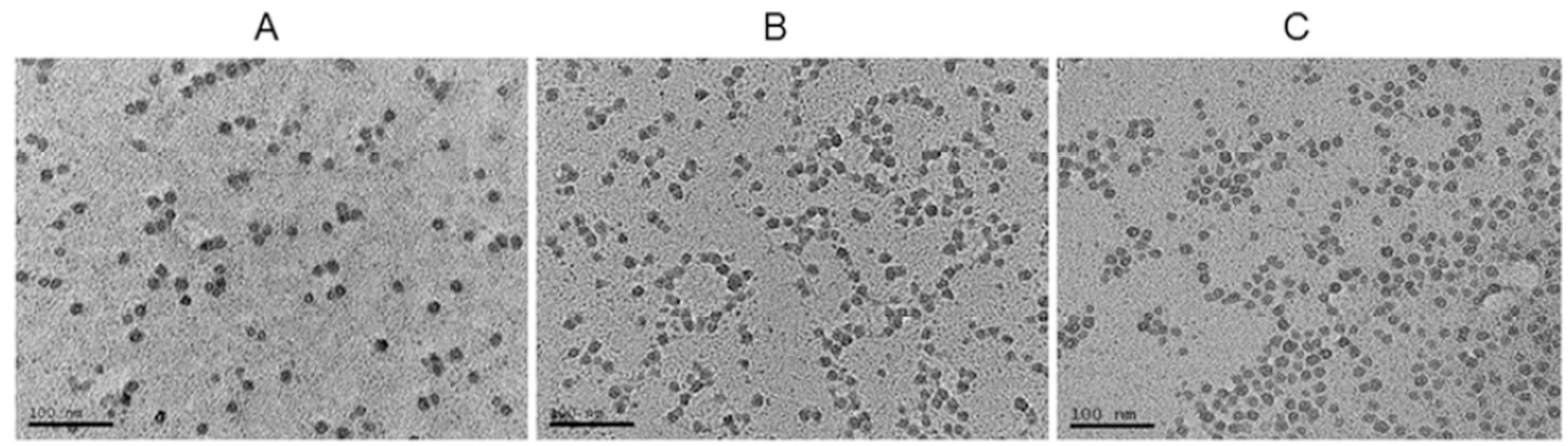

Figure 1.

TEM pictures of (A) cMyc-LCPs, (B) GMP-LCPs and (C) $($ GMP+cMyc)-LCPs. Scale bar = $100 \mathrm{~nm}$. 
A

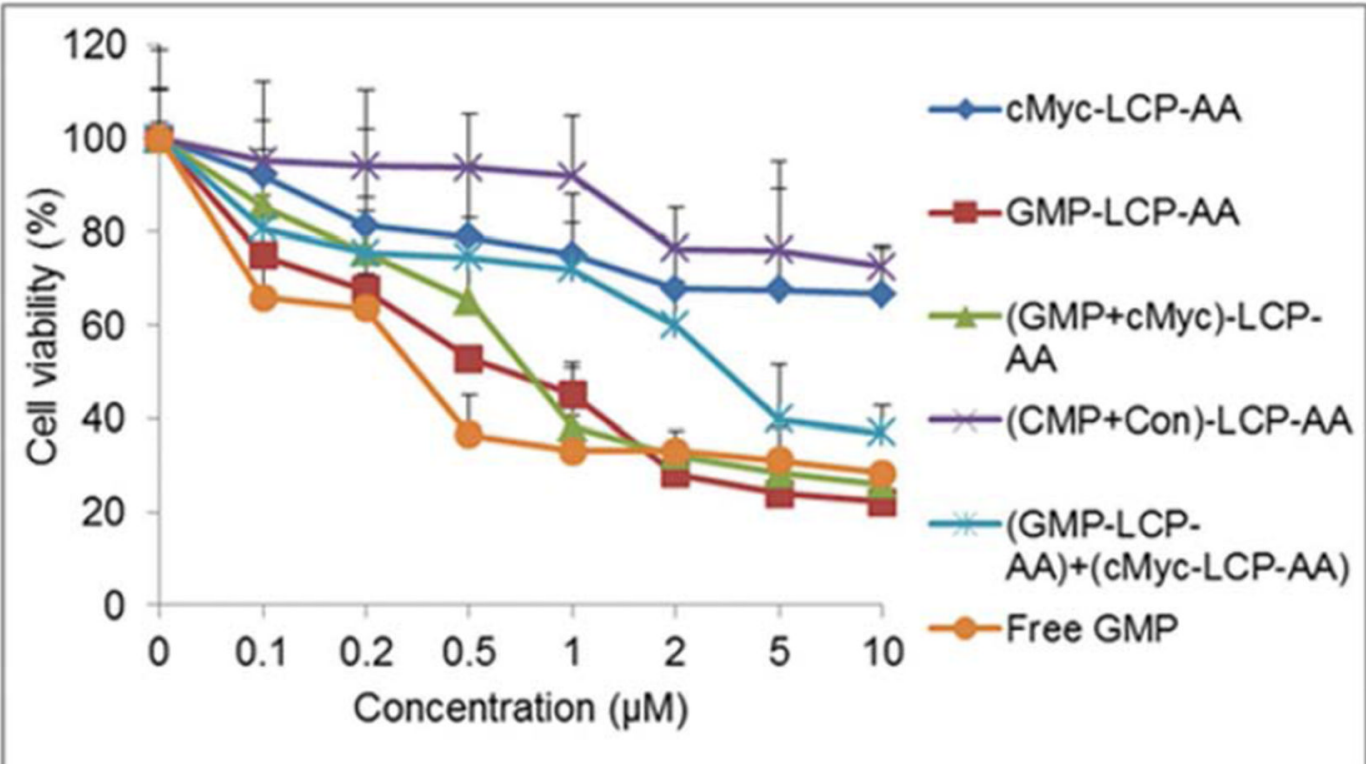

B

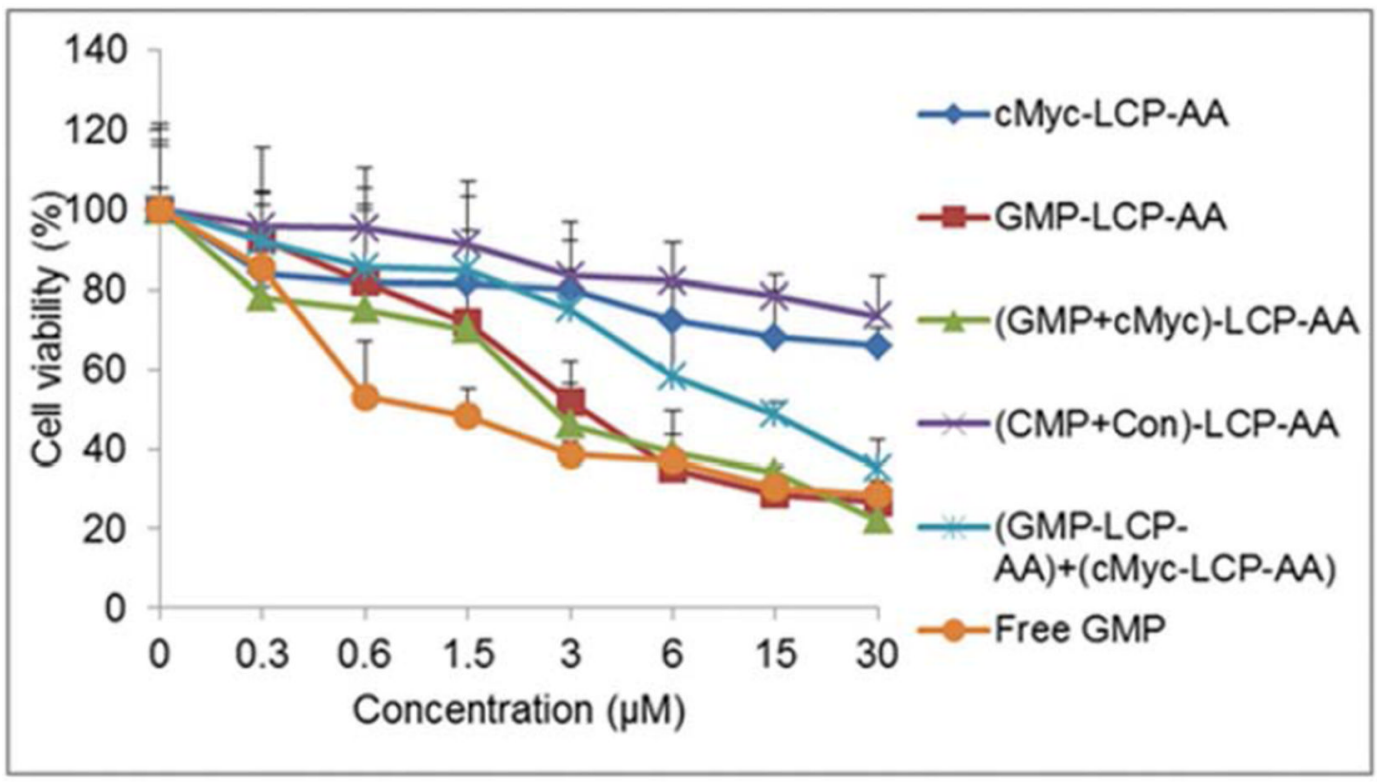

Figure 2.

Drug-loaded LCPs induced potent cytotoxicity in vitro on (A) H460 and (B) A549 cells. The cell viabilities of (A) H460 and (B) A549 cells were measured using an MTT assay after 48 $\mathrm{h}$ of exposure to cMyc-LCP-AA, GMP-LCP-AA, (GMP+cMyc)-LCP-AA, (CMP+Con)LCP-AA, free GMP, and a GMP-LCP-AA and cMyc-LCP-AA mixture (each at half dose). Data are mean \pm S.D. $(n=3)$. 


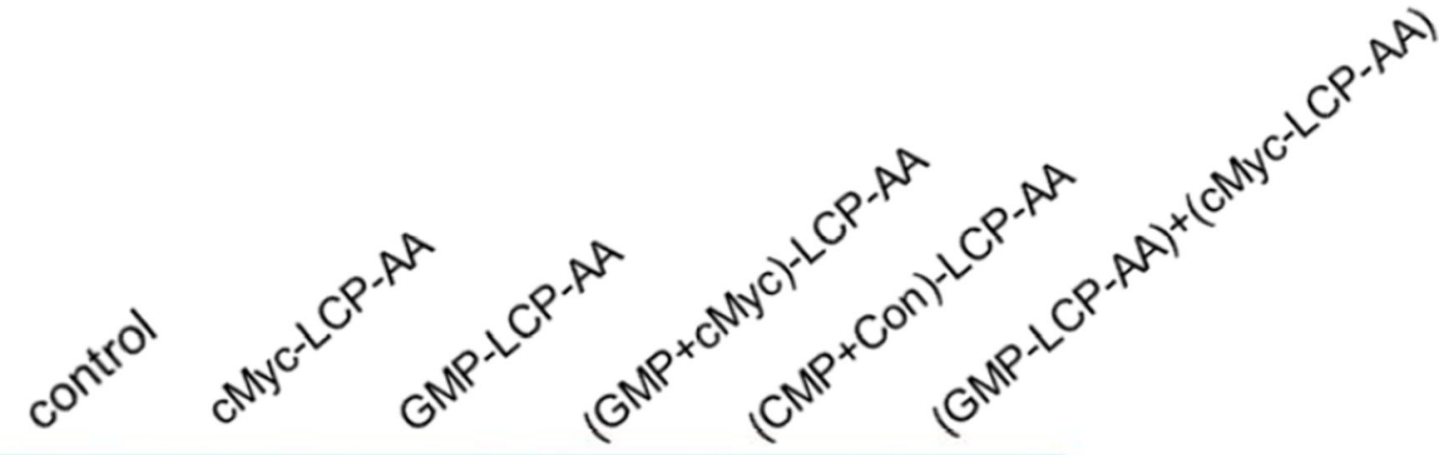

c-Myc

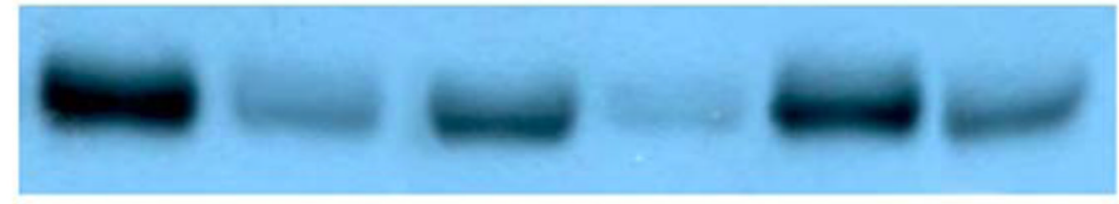

PARP

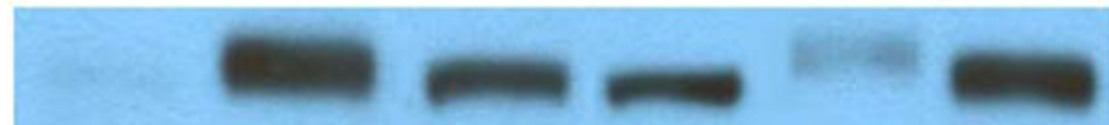

Cleaved PARP

ß-actin

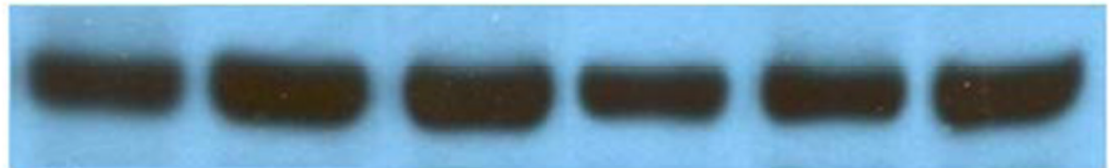

Figure 3.

Analysis of tumor proteins after systemic treatments. Mice bearing H460 tumors were given three daily IV injections and analysis of tumor proteins was prepared $24 \mathrm{~h}$ after the final injection using a western blot technique. 

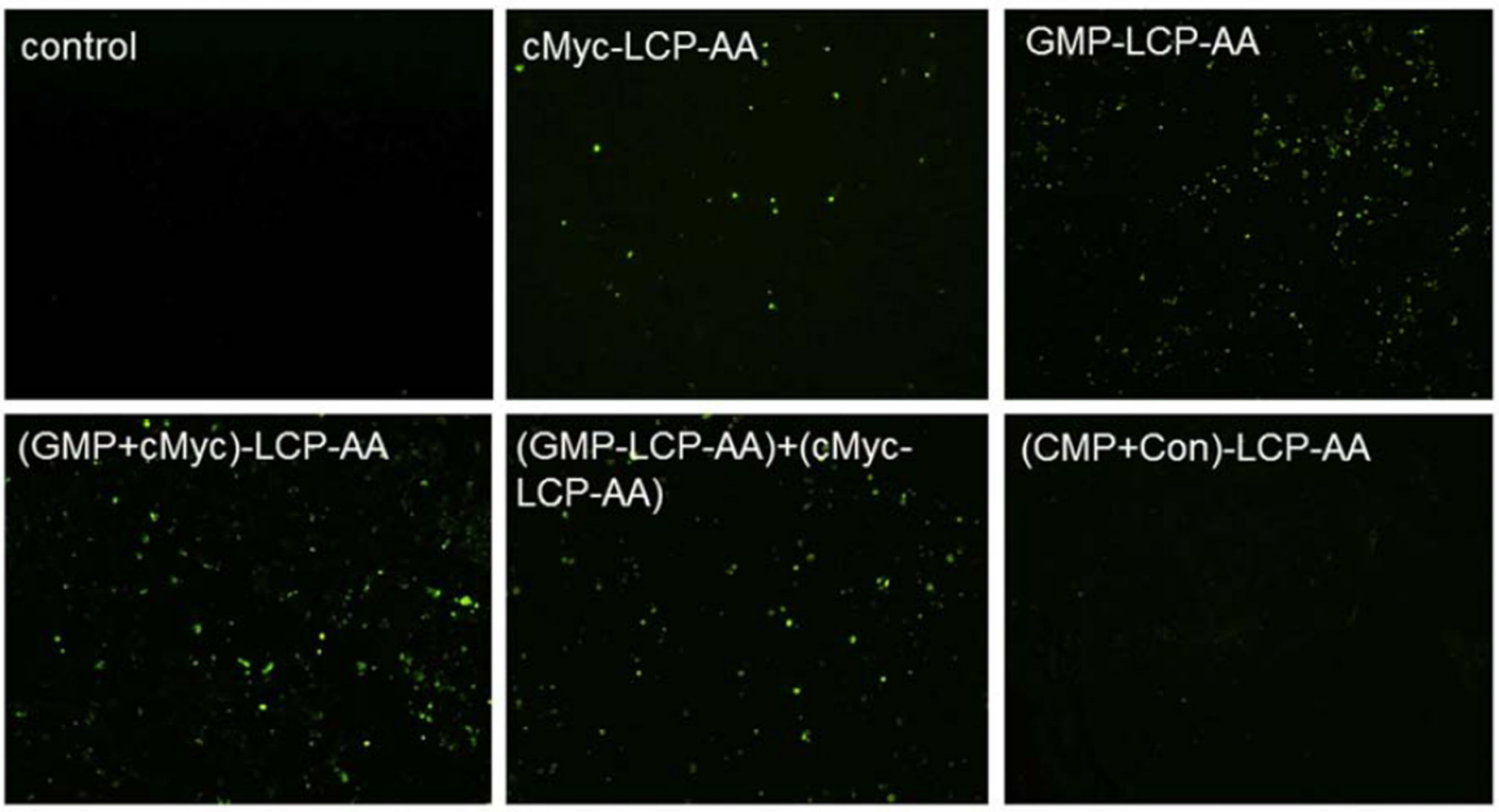

(CMP+Con)-LCP-AA 


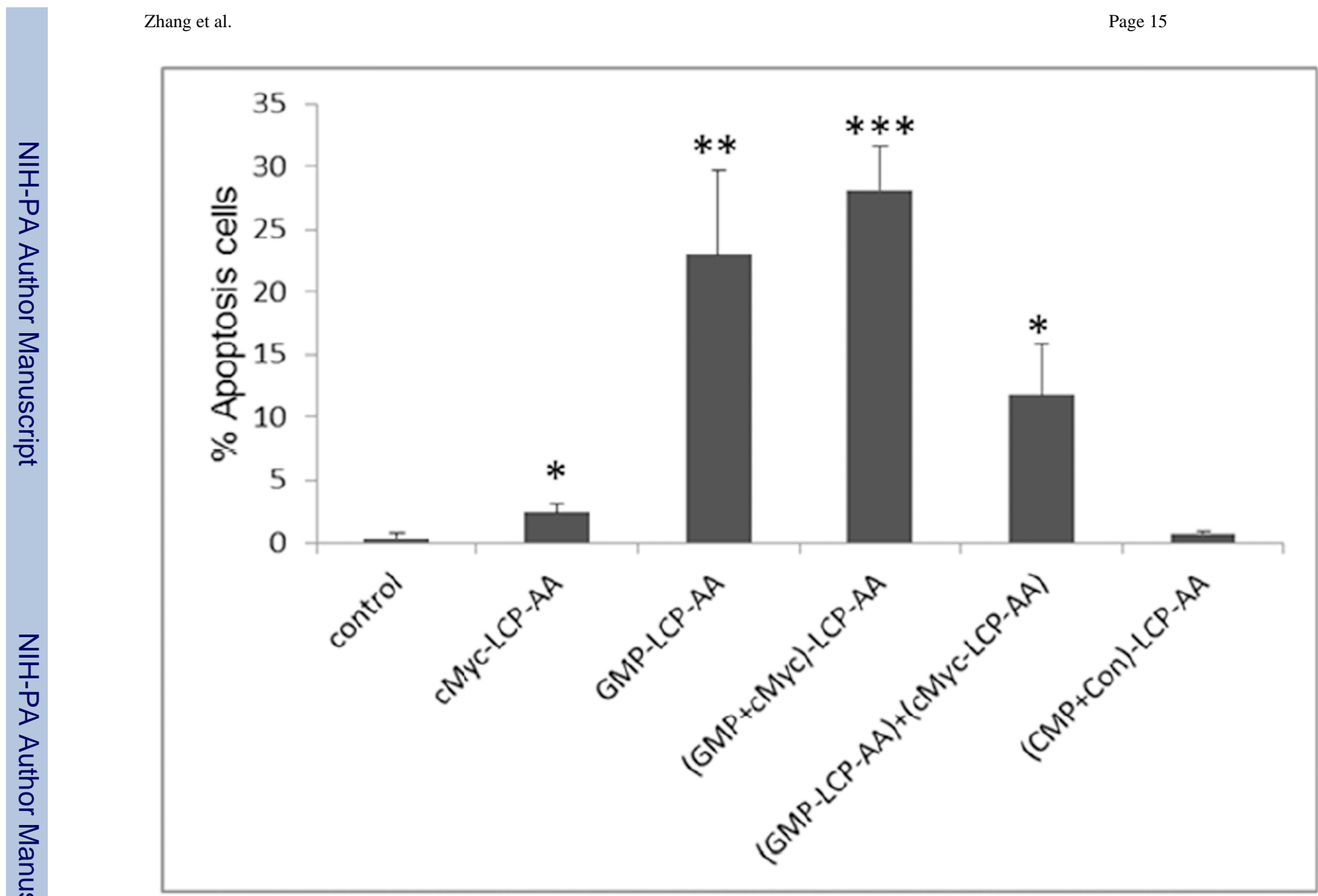



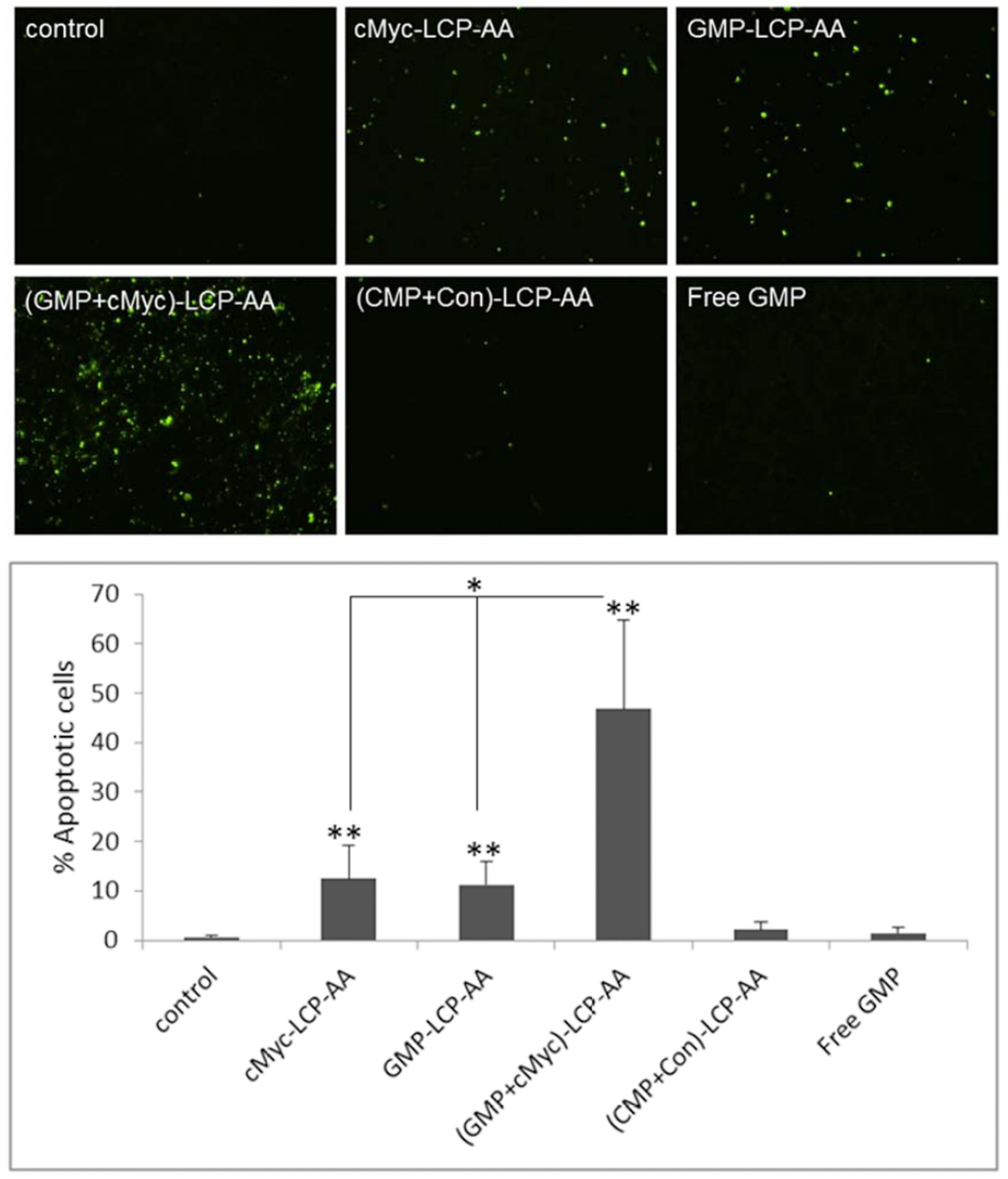

Figure 4. 
Apoptosis of tumor cells induced in vivo after the systemic administration of different LCPs in (A, B) H460 subcutaneous xenografts and (C, D) A549 orthotopic xenografts. The percentage (\%) of apoptotic cells in (B) H460 and (D) A549 xenograft models: (B) * $p<0.01$, cMyc-LCP-AA vs. control, (GMP-LCP-AA)+(cMyc-LCP-AA) vs. control, (GMP-LCP-AA) $+(\mathrm{cMyc}-\mathrm{LCP}-\mathrm{AA}) v s$. cMyc-LCP-AA; $* * p<0.001$, GMP-LCP-AA $v s$. control, GMP-LCPAA vs. cMyc- LCP-AA; *** $p<0.0001,(\mathrm{GMP}+\mathrm{cMyc})-\mathrm{LCP}-\mathrm{AA} v s$. control, (GMP+cMyc)LCP-AA $v s$. cMyc-LCP-AA; (D) * $p<0.0001$, cMyc-LCP-AA $v s$. (GMP+cMyc)-LCP-AA, GMP-LCP-AA $v s .(\mathrm{GMP}+\mathrm{cMyc})-\mathrm{LCP}-\mathrm{AA} ; * * p<0.00001$, cMyc-LCP-AA $v s$. control, GMP-LCP-AA vs. control, (GMP+cMyc)-LCP-AA vs. control. ( $\mathrm{n}=5$ per group) 

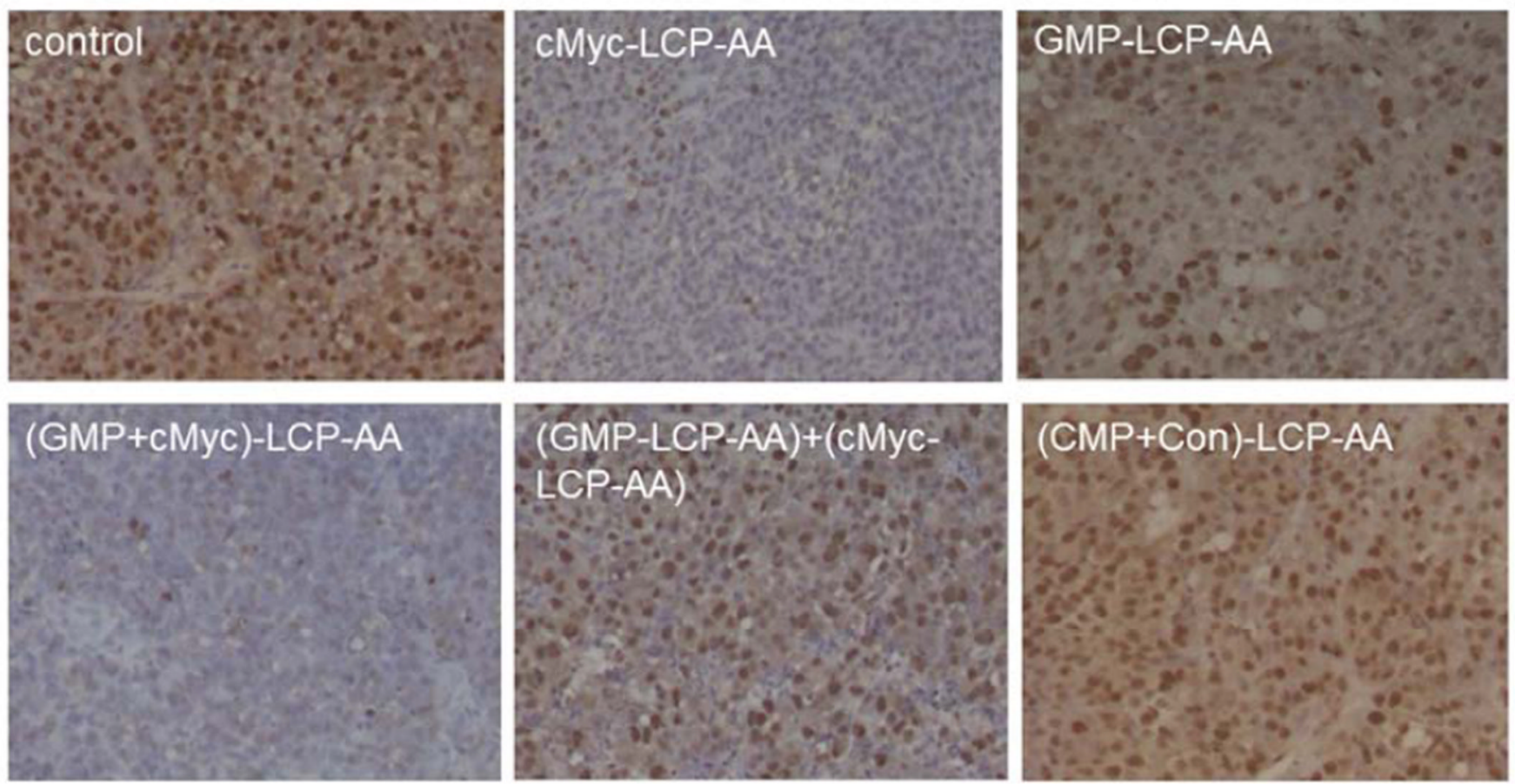


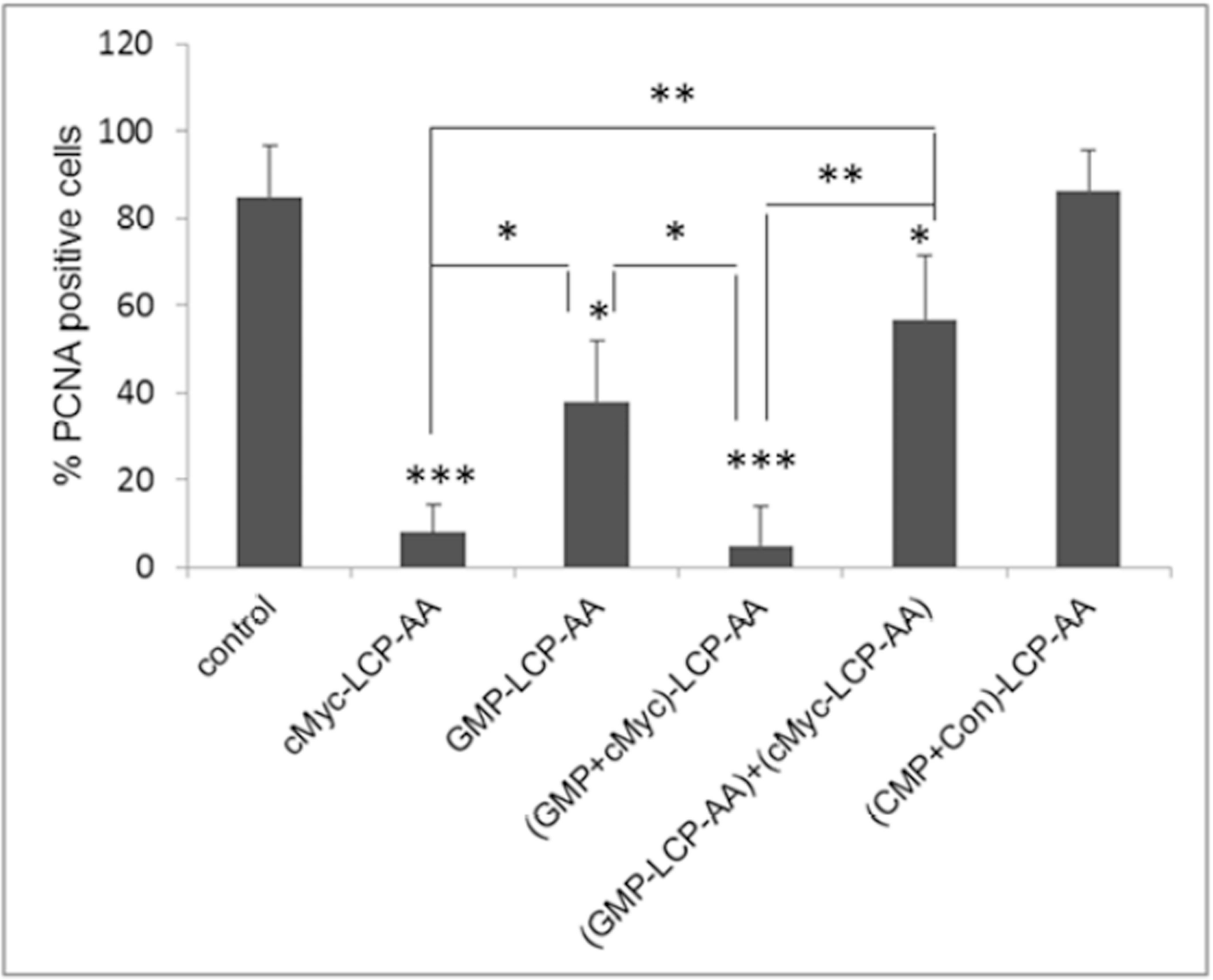

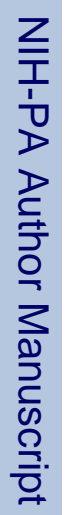



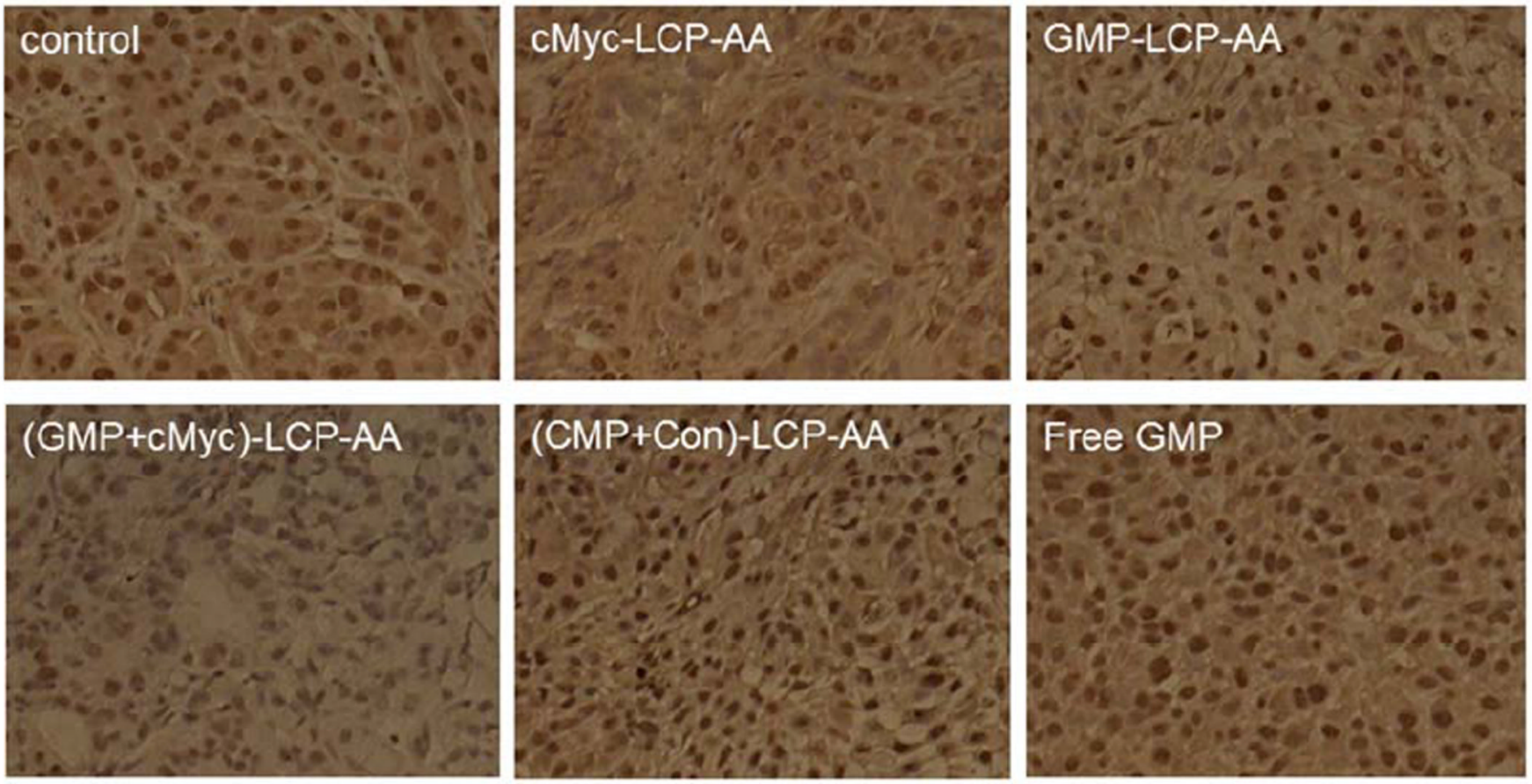

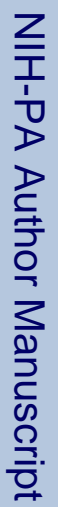

Biomaterials. Author manuscript; available in PMC 2014 November 01. 


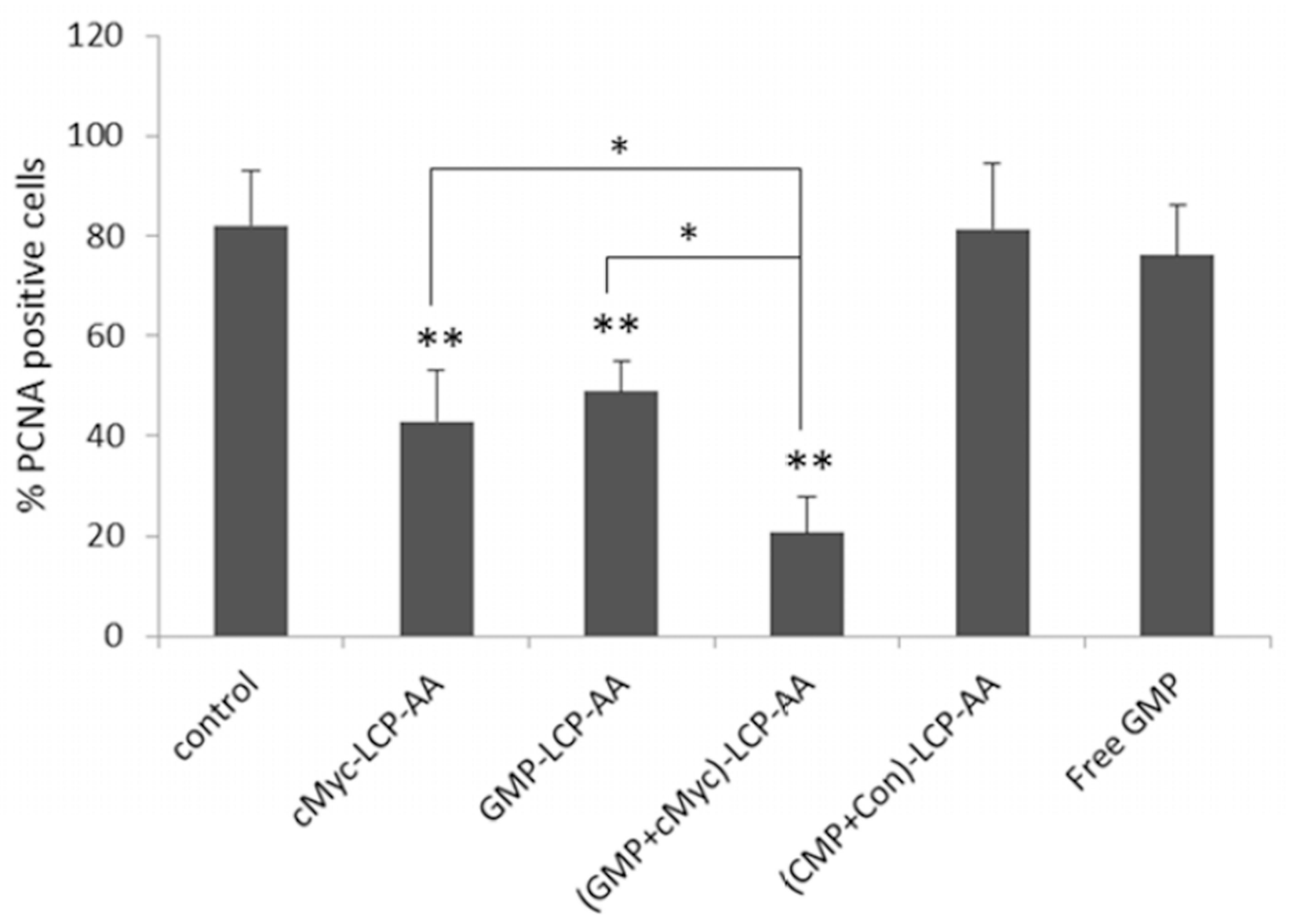

Figure 5.

Proliferation of tumor cells in vivo after administration of different LCPs in (A, B) H460 subcutaneous xenografts and (C, D) A549 orthotopic xenografts. Twenty-four h after 3 daily IV injections, mice were sacrificed and tumors tissues were sectioned for PCNA immunohistochemistry. The percentage (\%) of PCNA positive cells in (B) H460 and (D) A549 xenograft models was shown as follows: (B) *p<0.05, GMP-LCP-AA vs. control, GMP-LCP-AA $v s$. cMyc-LCP-AA, GMP-LCP-AA $v s$. (GMP+cMyc)-LCP-AA; ** $p<0.01$, cMyc-LCP-AA vs. (GMP-LCP-AA)+(cMyc-LCPAA), (GMP+cMyc)-LCP-AA vs. (GMPLCP-AA)+(cMyc-LCP-AA); $* * * p<0.001$, cMyc-LCP-AA vs. control, (GMP+cMyc)-LCPAA $v s$. control; (D) *p<0.01, cMyc-LCP-AA $v s$. (GMP+cMyc)-LCP-AA, GMP-LCP-AA $v s .(\mathrm{GMP}+\mathrm{cMyc})-\mathrm{LCP}-\mathrm{AA} ; * * p<0.0001$, cMyc-LCP-AA vs. control, GMP-LCP-AA $v s$. control, (GMP+cMyc)-LCP-AA vs. control. ( $\mathrm{n}=5$ per group) 


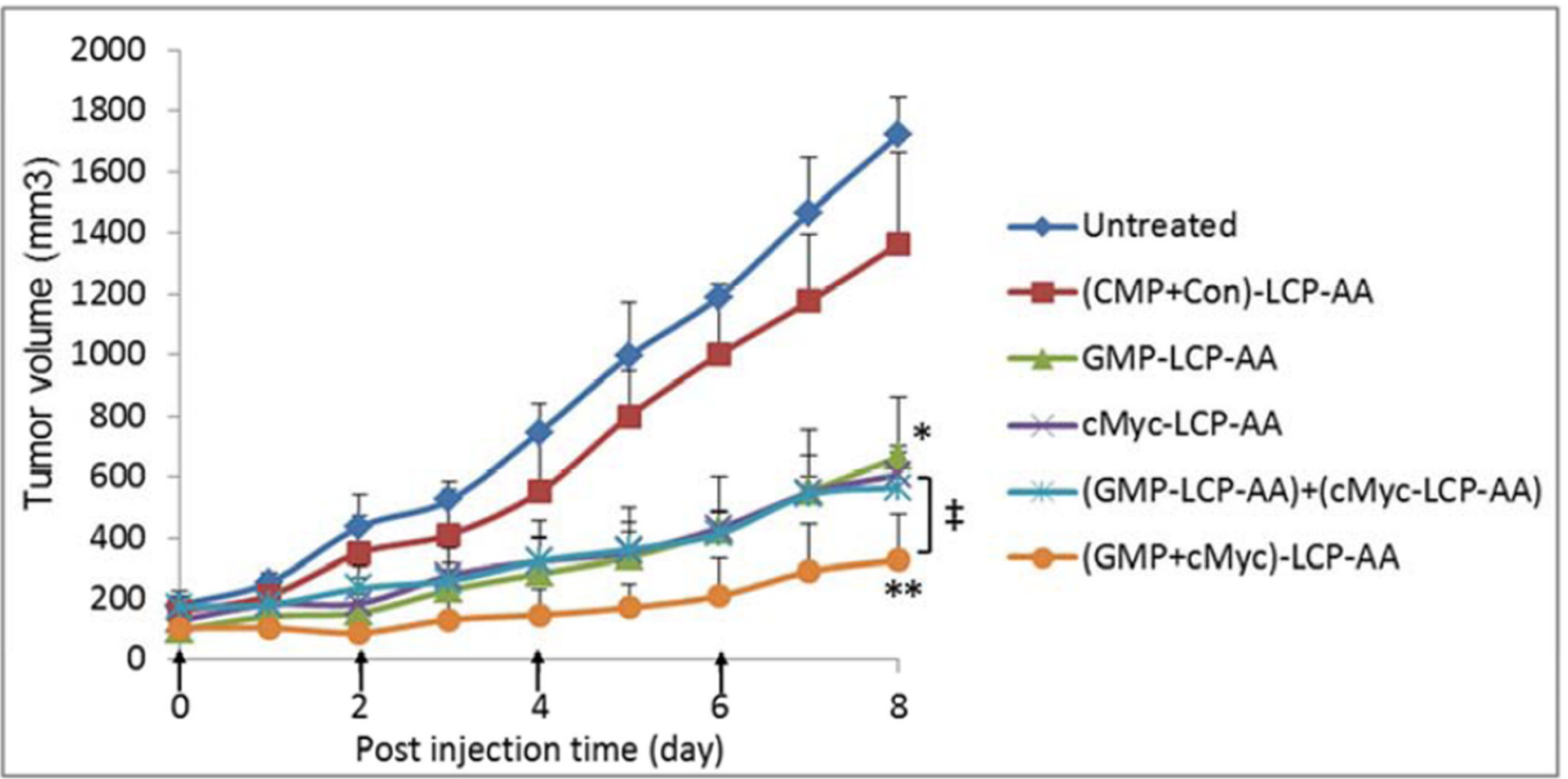



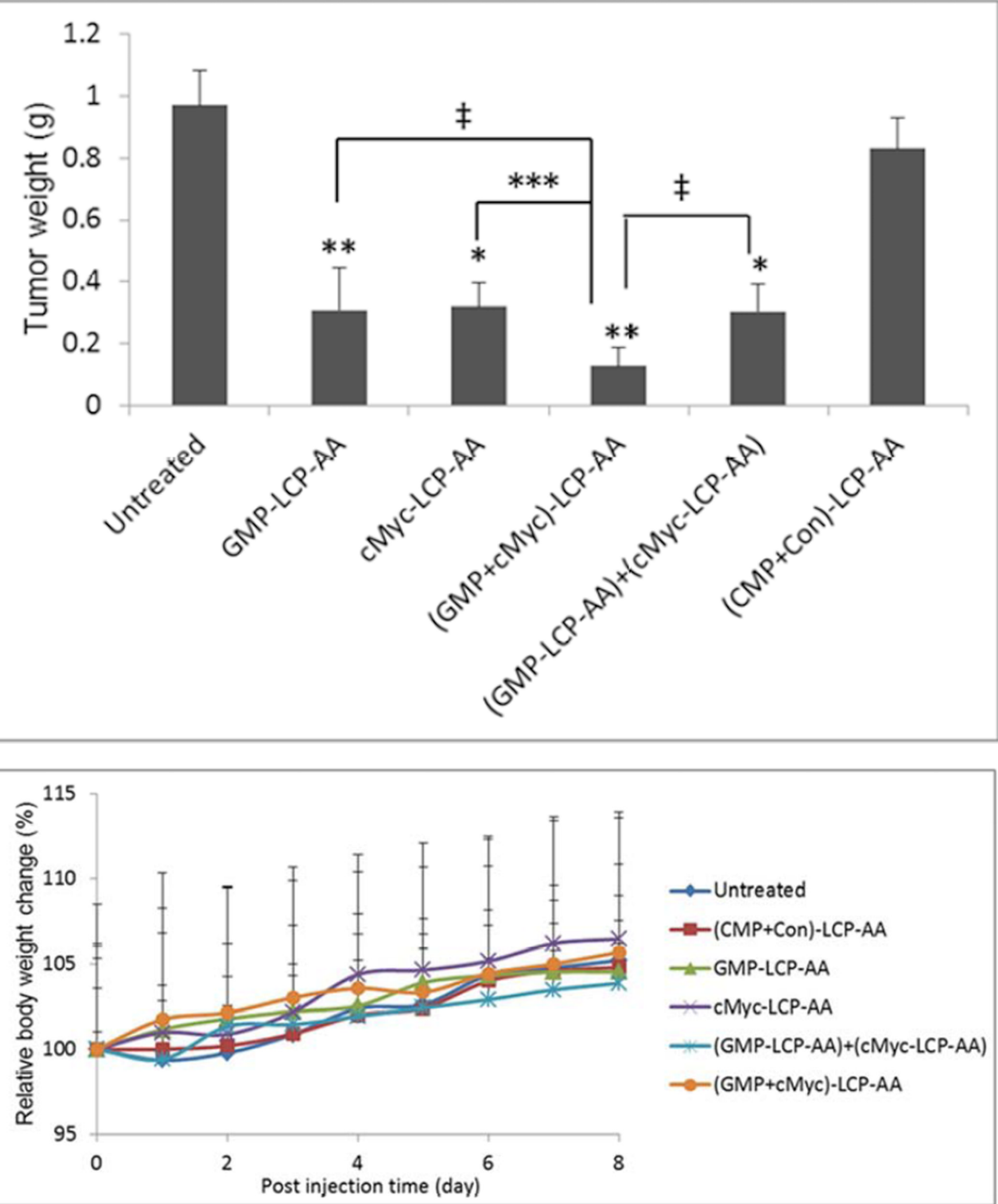

Figure 6. 
Inhibition of tumor growth in H460, subcutaneous xenografts of NSCLC. (A) Inhibition of tumor growth by different LCP formulations on H460-tumor bearing mice. cMyc-LCP-AA, GMP-LCP-AA, (GMP+cMyc)-LCP-AA, (CMP+Con)-LCP-AA and free GMP were administered intravenously every other day for total 4 injections. Tumor volumes were measured every other day. Data are mean \pm S.D. $(\mathrm{n}=5)$ Statistics are as follows: $*_{p}<0.0005$, GMPLCP- AA vs. control, cMyc-LCP-AA vs. control, (GMP-LCP-AA)+(cMyc-LCP-AA) vs. control; $* * p<0.00001$, (GMP+cMyc)-LCP-AA vs. control; $\ddagger \mathrm{p}<0.05$, GMP-LCP-AA vs. (GMP+cMyc)-LCP-AA, cMyc-LCP-AA vs. (GMP+cMyc)-LCP-AA, (GMP-LCP-AA)+ (cMyc-LCP-AA) vs. (GMP+cMyc)-LCP-AA. (B) Weights of tumors collected from treated mice. Data are expressed as mean \pm S.D. $(\mathrm{n}=5)$ Statistics are as follows: ${ }^{*} p<0.005$, cMycLCP-AA vs. control, (GMP-LCP-AA)+(cMyc-LCP-AA) vs. control; **p $<0.001$, GMPLCP-AA vs. control, (GMP+cMyc)-LCP-AA vs. control; ***p<0.01, cMyc-LCP-AA vs. (GMP+cMyc)-LCP-AA; $\neq \mathrm{p}<0.05$, GMP-LCP-AA vs. (GMP+cMyc)-LCP-AA, (GMP-LCPAA)+(cMyc-LCP-AA) vs. (GMP+cMyc)-LCP-AA. (C) The relative body weight changes over the treatment period of mice bearing H460 tumors. Data are mean \pm S.D. $(n=5)$ 


\section{Table 1}

Serum levels of BUN, creatinine, AST, ALT after three daily IV injections in BALB/c mice.

\begin{tabular}{ccccc}
\hline & BUN mg/dL & $\begin{array}{c}\text { Creatinine } \\
\mathbf{~ m g / d L}\end{array}$ & AST U/L & ALT U/L \\
\hline Control & $26.0 \pm 3.0$ & 0.2 & $140.0 \pm 5.0$ & $46.0 \pm 2.0$ \\
GMP-LCP-AA & $27.3 \pm 6.0$ & 0.2 & $134.3 \pm 14.2$ & $47.7 \pm 6.1$ \\
cMyc-LCP-AA & $22.3 \pm 3.5$ & 0.2 & $144.3 \pm 37.6$ & $56.7 \pm 12.5$ \\
(GMP+cMyc)-LCP-AA & $23.6 \pm 4.0$ & 0.2 & $130.7 \pm 26.6$ & $54.7 \pm 5.5$ \\
(GMP-LCP-AA)+(cMyc-LCP-AA) & $23.8 \pm 4.1$ & 0.2 & $123.0 \pm 22.0$ & $53.8 \pm 8.3$ \\
(CMP+Con)-LCP-AA & $25.0 \pm 3.4$ & 0.2 & $141 \pm 10.2$ & $53.0 \pm 4.1$ \\
\hline Reference Range & $8--33$ & $0.2--0.9$ & $54--298$ & $17--132$ \\
\hline
\end{tabular}

